\title{
Development of a Mode Choice Model for Bus Rapid Transit in Santa Clara County, California
}

\author{
Chun-Hung Peter Chen and George A. Naylor \\ Santa Clara Valley Transportation Authority
}

\begin{abstract}
Bus Rapid Transit (BRT) is an enhanced bus service that offers many of the same service attributes as rail transit, such as specialized vehicles, large stations, real-time passenger information, and more frequent and reliable operations. The Santa Clara Valley Transportation Authority (VTA) intends to develop an integrated BRT network throughout Santa Clara County, California, to provide high quality service to areas not well served by the VTA Light Rail (LRT) system. Past research showed that many transit agencies in North America considered BRT the same as LRT in their demand models, and a few agencies treated BRT and local bus identically. Realistic $B R T$ ridership forecasts are essential for selecting and sizing facilities, preparing service plans, estimating capital and operating costs, and assessing cost-effectiveness. This study applied the results of the transit preference survey in a Market Research Model prepared for the VTA and built the improved mode choice model that explicitly included the BRT mode in the VTA demand model. Instead of considering BRT the same as either LRT or local bus, the improved VTA model with an explicit BRT mode is expected to forecast more reasonable future BRT boardings. Eleven scenarios in the BRT strategic plan for Santa Clara County were developed using the BRT forecast results from the improved VTA model.
\end{abstract}




\section{Introduction}

Bus Rapid Transit (BRT) is an enhanced bus service that offers many of the same service attributes as rail transit, such as specialized vehicles, large stations, real-time passenger information, and more frequent and reliable operations. A more detailed definition developed by the Transit Cooperative Research Program (TCRP) as part of TCRP Report 90 (2003) is that "BRT is flexible, rubber-tired rapid transit mode that combines stations, vehicles services, running ways, and Intelligent Transportation System (ITS) elements into an integrated system with a strong positive identity that evokes a unique image ... In brief, BRT is an integrated system of facilities, services, and amenities that collectively improves the speed, reliability, and identity of bus transit."

Vuchic (2002) defined BRT based on combining mode performance (speed, reliability, capacity, image) and investment cost per kilometer of line for three categories of transit modes-rapid transit (Metro), semi-rapid transit (light rail transit, LRT), and street transit (regular bus) - and expresses the definition of BRT as the transit mode between LRT and regular bus. Levinson et al. (2002) proposed the comparisons of BRT and other transit modes as follows: "1. where BRT vehicles (buses) operate totally on exclusive or protected rights-of-way, the level of service provided can be similar to that of full Metrorail rapid transit; 2 . where buses operate in combinations of exclusive rights-of-way, median reservations, bus lanes, and street running, the level of service provided is very similar to LRT; 3 . where buses operate mainly on city streets in mixed traffic, the level of service provided is similar to a limited-stop tram/streetcar system." In general, BRT operating in combinations of exclusive bus lane and mixed traffic is considered to be a transit mode between LRT and local bus.

BRT is now a major trend in the development of public transportation systems worldwide. In the U.S., several BRT systems are in service, such as in Eugene (Oregon), Los Angeles, and Cleveland, and there are also other BRT systems under construction, in development, or planned. According to a Federal Transit Administration's study (2005), in areas with new BRT systems, about 24 to 33 percent of $B R T$ ridership is new to transit. BRT ridership-and transit ridership forecasting in general-is an integral part of transportation planning. Realistic estimates of BRT ridership are essential for selecting and sizing facilities, preparing service plans, estimating capital and operating costs, qualifying benefits, and assessing costeffectiveness (TCRP 2006). TCRP (2006) implemented BRT ridership surveys for 20 transit agencies in North America to ascertain how BRT was treated in their travel 
demand forecasting. This study found many agencies considered BRT the same as LRT in their demand models, and only a few agencies treated BRT and local bus identically. It was also found that no transit agencies had built new specific BRT modes in their models for analyzing BRT in the study survey.

The Santa Clara Valley Transportation Authority (VTA) intends to develop an integrated BRT network throughout Santa Clara County, California, to provide high quality service to the areas not served by LRT. VTA has developed the Santa Clara County BRT Strategic Plan (2009) in which different BRT alternatives, potential corridors, operating and infrastructure strategies were proposed. Near-term and long-term BRT corridors integrated with the existing transit system and road system within the county, including Caltrain, LRT, bus, and exclusive lanes with signal priority, will provide the community with more comprehensive and convenient transit service. Future BRT ridership forecasting is one critical element for BRT planning. The current VTA countywide model does not include a BRT mode in the mode choice model. Based on the current structure of the VTA models, if BRT is considered the same as LRT, the forecast ridership may be overestimated. Conversely, if BRT is considered the same as a local bus, the forecast ridership may be underestimated. Given the anticipated need for the level of detail required in developing future BRT plans, it was necessary for the VTA to develop a refined mode choice model that included the mode of BRT.

The purpose of this study was to develop an enhanced mode choice model including the mode of BRT into the VTA model so that the model can forecast future BRT ridership for the planning, development, and implementation of the BRT system in Santa Clara County. The model proposed in this study also is used for alternatives analysis, prioritizing BRT corridors, analysis of new transit trips, and examining impacts to background local bus services. The "previous model" used in this paper represents the original VTA countywide model without applying the procedures of the BRT mode choice model developed in this study; the "improved model" represents the revised model using the new BRT mode choice model.

\section{Previous VTA Model}

VTA has developed and maintained a countywide travel demand model for at least a decade, which has been applied to various countywide transportation planning and engineering projects. The VTA model initially was structured to be consistent with the Metropolitan Transportation Commission (MTC) regional model, BAY- 
CAST (1997). MTC is the metropolitan planning organization (MPO) for the ninecounty San Francisco Bay area. The VTA countywide model is an enhanced version of the MTC nine-county regional model, with the addition of more traffic analysis zones (TAZs) and more detailed highway and transit network coding within Santa Clara County. The MTC mode choice model also was enhanced for application in Santa Clara County and the greater modeling region. In the original MTC model, trips were first split into motorized modes and bicycle and walk-only modes. Motorized trips were then split into drive alone, shared ride 2, shared ride 3 plus, and transit. Last, transit trips were split into transit walk access versus transit auto access. All transit modes were treated identically in the MTC mode choice model, and the choice as to whether the trip used heavy rail, commuter rail, light rail, or express or local bus was dependent on the shortest time path. The enhancements from the MTC model to the VTA model included the implementation of a transit submode nest, allowing the models to estimate ridership on the different transit submodes of commuter rail, express bus, local bus, BART (heavy rail), and light rail as distinct choices based on relative costs and travel times that occur for each submode. The constants of the utility functions for commuter rail, express bus, local bus, BART (heavy rail), and light rail were calibrated based on the transit onboard survey data and transit boarding data. With the inclusion of distinct transit submodes as choices in the model structure, it was possible to calibrate mode specific constants in the VTA mode choice models for each submode. Typically, submode specific constants capture the importance of modal attributes not typically included in the mode choice utility equations, such as reliability, passenger comfort, and safety. During base year calibration, for home-based work trips, the addition of transit submode constants improved the level of validation for each submode. Home-based work calibration results yielded a less negative constant on light rail, followed by heavy rail, commuter rail, local bus, and express bus, in that order. This implies that, all things being equal with respect to travel times and costs, there is a higher probability that a trip will use rail over bus. For the non-work purposes, transit submodes behave in a much more generic manner, with only slight biases for rail in the home-based shop/other and home-based social recreational models. The exception in the non-work models was with the non-home-based trip purposes, as both heavy rail and light rail were shown to have less negative constants as compared to commuter rail or bus modes. Figure 1 without the dashed line box shows the mode choice structure at the previous VTA model. 


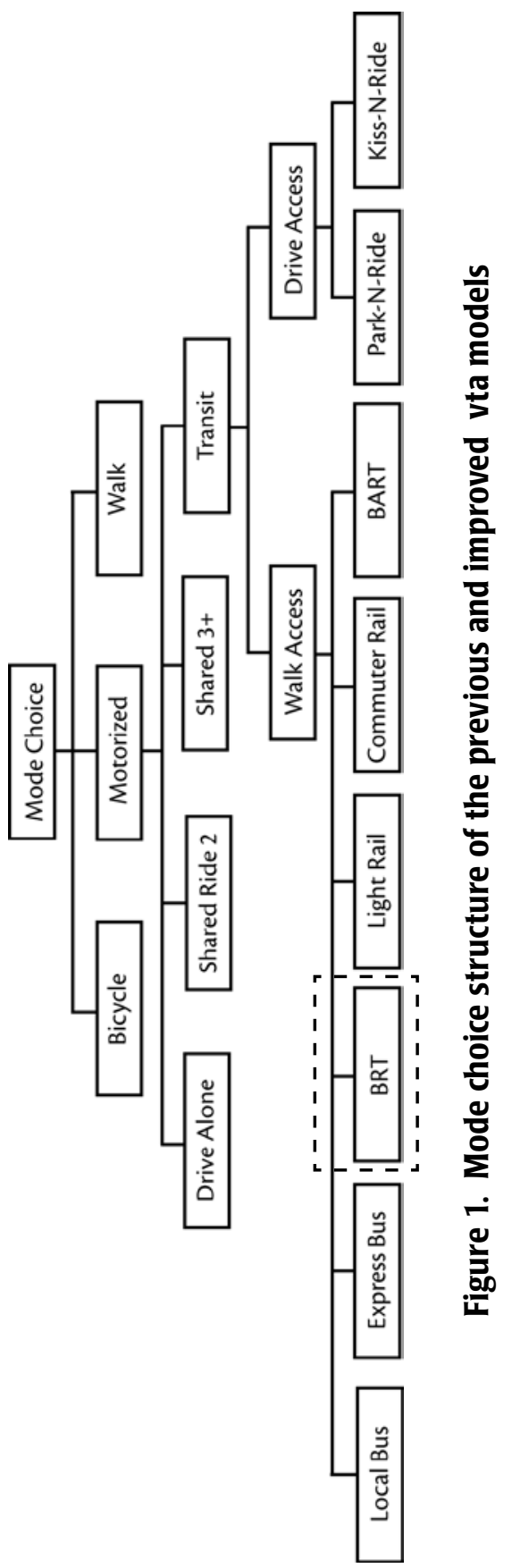




\section{Improved VTA Model}

The BRT mode was added into the VTA mode choice model for developing the BRT ridership forecasts to support the Santa Clara County BRT strategic plan. Figure 1 with the dashed line box of the BRT mode shows the mode choice structure of the improved VTA model. The important parameters used in the improved VTA mode choice model, i.e., BRT constants, were derived from the Transit Market Research Model (2007) developed for the VTA. This section addresses how the BRT mode was developed by applying the Transit Market Research Model into the VTA demand model while BRT was still in development and planned without any observed BRT operating data.

\section{Transit Market Research Model}

VTA developed a transit market research project, implemented by Cambridge Systematics, Inc., to support the Comprehensive Operational Analysis (COA), a major service redesign plan for the entire VTA bus system that was implemented in January 2008. Transit market research is used to develop market segments based on travelers' attitude towards everyday transportation experiences. The VTA transit market research project consisted of three distinct tasks: data collection, attitudinal-based market segmentation modeling, and mode choice modeling. Data collection included a stated-preference survey of 819 households throughout Santa Clara County. The survey collected attitudinal, demographic, and travel behavior data. The attitudinal-based market segmentation uses cluster analysis techniques to group individual travelers according to their attitudes toward transportation to identify market segments, and then expands the survey records to the entire population of Santa Clara County.

The importance of Transit Market Research Model introduced here is because a new mode of travel-BRT-was estimated in the market research mode choice models. Market research-based mode choice models were developed with the data collected from the market research household travel surveys, specifically from four customized mode choice experiments. Four experiments in the surveys have different values of time, costs, and amenities. Three transit service amenities to address packages of BRT and other transit modes include an electronic sign showing minutes until next train, distinctive-looking buses with comfortable interior, and well-lit, covered stations equipped with benches, maps, and guides. Because BRT was not in service currently, through attitudinal and stated preference surveys, the ridership of BRT likely transferred from current transit systems and potential new ridership from auto modes could be estimated by the market research-based mode 
choice models. The market research-based mode choice models are multinomial logit models for work and non-work trip purposes. The results of the mode choice models, including the coefficients of different variables in the utility functions and the bias constants for each transit mode (rail, BRT, and bus) are shown in Table 1.

Table 1. Market Research-Based Mode Choice Models

\begin{tabular}{|c|c|c|c|}
\hline Categories & Variables & $\begin{array}{c}\text { Home-Based } \\
\text { Work/University }\end{array}$ & Non-Work \\
\hline IVTT & In-Vehicle Travel Time & -0.0330 & -0.0091 \\
\hline \multirow[t]{5}{*}{ OVTT } & Walk time-Access/Egress & -0.0650 & -0.0233 \\
\hline & Wait time $<=7$ mins & -0.0650 & -0.0233 \\
\hline & Wait time $>7$ mins & -0.0500 & -0.0179 \\
\hline & Drive-Access Time & -0.0650 & -0.0233 \\
\hline & Transfer Time & -0.0650 & -0.0233 \\
\hline Cost & Cost & -0.0770 & -0.0718 \\
\hline \multirow[t]{3}{*}{ Attitudinal Factors } & Pro-environment & 0.5750 & - \\
\hline & Social Perception & -0.2430 & -0.5512 \\
\hline & Travel Flexibility & -0.1450 & - \\
\hline \multirow{6}{*}{$\begin{array}{l}\text { Social-Economic } \\
\text { Variable }\end{array}$} & Workers/ Household & -0.0630 & - \\
\hline & Vehicle/ Household & 0.0000 & -0.0670 \\
\hline & Age 18 to 24 & 1.5180 & 1.8589 \\
\hline & Income $<\$ 25,000$ & 1.0360 & 1.4565 \\
\hline & Income $\$ 25,000$ to $\$ 50,000$ & 0.2520 & -0.2244 \\
\hline & Female & -0.6210 & -0.3754 \\
\hline \multirow[t]{3}{*}{ Transit Amenities } & Amenities -Signs & 0.2140 & 0.5281 \\
\hline & Amenities -Buses & 0.2930 & 0.0187 \\
\hline & Amenities Stations & 0.4220 & 0.5100 \\
\hline \multirow[t]{4}{*}{ Modal Constants } & Drive Alone - base constant & 0.0000 & 0.0000 \\
\hline & LRT- constant & 0.0000 & -1.7593 \\
\hline & BRT - constant & -0.0340 & -1.8115 \\
\hline & Bus - constant & -0.7810 & -1.8025 \\
\hline \multirow[t]{3}{*}{ Perform Measures } & Value of Time & $\$ 25.37$ & $\$ 7.64$ \\
\hline & OVTT(wait time <= 7 mins) /IVTT & 2.0 & 2.6 \\
\hline & OVTT(wait time> 7 mins) /IVTT & 1.5 & 2.0 \\
\hline
\end{tabular}

Note: OVTT: out-vehicle travel time; IVTT: in-vehicle travel time

Source: Santa Clara Valley Transportation Authority, 2007. 


\section{Translation of BRT Constants}

Though the purpose of the market research project was to support the transit comprehensive operational analysis, and the market research-based mode choice models were not directly applied in the VTA demand model, the bias constants of BRT compared to (light) rail and bus can be applied to add the new BRT mode in the VTA demand model. Constant coefficients can be converted into bias time constants by dividing constant coefficient by in-vehicle time coefficient

$$
b_{m}=\frac{c_{m}}{c_{i v t}}
$$

where $b_{m}$ is bias time constant for mode $m ; c_{m}$ is constant coefficient for mode $m$ and $c_{\text {ivt }}$ is in-vehicle time coefficient in Market Research Model. Bias time constants present the relative waiting time among different transit modes. For home-based work trips, the rail, $\mathrm{BRT}$, and bus constants are $0,-0.034$, and -0.781 . Using Eq. (1), the bias time constants for rail, $B R T$, and bus are $0,-1.03$ and -23.67 minutes, respectively. For non-work trips, the rail, BRT, and bus constants are $-1.7593,-1.8115$, and -1.8025 . The bias time constants for rail, BRT, and bus converted to equivalent minutes of in-vehicle travel time are $-193.33,-199.07$ and -198.08 minutes, respectively. Due to home-based work passengers having a higher value of time at $\$ 25.37$ compared to non-work passengers' value of time at $\$ 7.64$, potential BRT passengers from homebased work trips consider BRT more like LRT, while non-work passengers consider BRT more like local bus. For home-based work passengers, BRT only provides one less minute travel time than light rail and 23 minutes travel time over local bus; for non-work passengers, BRT and local bus almost have no significant difference for equivalent time, -199.07 and -198.08 minutes. It was, therefore, assumed that BRT and local bus have the same bias time constants for non-work trips.

Bias time constants derived from Transit Market Model were used to estimate the BRT constants in the VTA demand model. Table 2 shows the coefficients of utility functions of the previous VTA mode choice model without BRT constants. Because the BRT mode is considered to be service between that provided by light rail and local bus, BRT constants are calculated by the linear interpolation method using the light rail constants, local bus constants, and bias time constants obtained above.

$$
\Delta_{B R T}=\Delta_{L B}+\left(\Delta_{L R T}-\Delta_{L B}\right)\left(\frac{b_{B R T}-b_{L B}}{b_{L R T}-b_{L B}}\right)
$$

where $\Delta_{B R T}$ is BRT constant; $\Delta_{L B}$ is local bus constant; $\Delta_{L R T}$ is LRT constants; $b_{B R T}$ is BRT bias time constant; $b_{L B}$ is local bus bias time constant; and $b_{L R T}$ is LRT bias time constant. 


\section{Table 2. VTA Mode Choice Models-Transit Walk Access}

\begin{tabular}{|c|c|c|c|c|c|c|c|}
\hline Variables & $\begin{array}{l}\text { Home- } \\
\text { Based } \\
\text { Work }\end{array}$ & $\begin{array}{c}\text { Home- } \\
\text { Based } \\
\text { Shop- } \\
\text { ping }\end{array}$ & $\begin{array}{c}\text { Home- } \\
\text { Based } \\
\text { Social/ } \\
\text { Recre- } \\
\text { ation }\end{array}$ & $\begin{array}{l}\text { Non- } \\
\text { Home } \\
\text { Based }\end{array}$ & $\begin{array}{l}\text { Home- } \\
\text { Based } \\
\text { School } \\
\text { (Grade } \\
\text { School) }\end{array}$ & $\begin{array}{l}\text { Home- } \\
\text { Based } \\
\text { School } \\
\text { (High } \\
\text { School) }\end{array}$ & $\begin{array}{l}\text { Home- } \\
\text { Based } \\
\text { School } \\
\text { (College) }\end{array}$ \\
\hline $\begin{array}{l}\text { BART } \\
\text { (heavy rail) }\end{array}$ & -0.86301 & 1.14089 & 2.48260 & 4.74364 & 0.59115 & 1.11067 & 0.76854 \\
\hline $\begin{array}{l}\text { Commuter } \\
\text { Rail }\end{array}$ & -0.86301 & 1.02982 & 2.22221 & 3.57032 & 0.59115 & 1.11067 & 0.76854 \\
\hline Light Rail & -0.96318 & 1.02982 & 2.22221 & 4.84000 & 0.59115 & 1.11067 & 0.76854 \\
\hline Express Bus & -1.84149 & 1.02982 & 2.22221 & 3.57032 & 0.59115 & 1.11067 & 0.76854 \\
\hline Local Bus & -1.70196 & 1.02982 & 2.22221 & 3.57032 & 0.59115 & 1.11067 & 0.76854 \\
\hline EMPD & 0.546100 & & & & & & \\
\hline Zero VHHD & 0.550100 & 3.2910 & & & & & \\
\hline VHH & & -0.3352 & -0.7475 & & & & \\
\hline РHн^3 & & & & & 0.004436 & & \\
\hline Rurali & & & & & 1.544 & & \\
\hline Total Time & & -0.05815 & & & & & \\
\hline IVT & -0.033260 & & -0.02745 & -0.03232 & -0.05855 & -0.03228 & -0.02731 \\
\hline Wait & -0.052330 & & & -0.07836 & & & \\
\hline Walk & -0.093050 & & & -0.07583 & & & \\
\hline Transfer & -0.033260 & & & & & & \\
\hline OVTT & & & -0.06806 & & -0.06384 & -0.03463 & -0.03923 \\
\hline Cost & -0.002067 & & & & & & \\
\hline LnCost & & -0.2262 & -1.1600 & -0.9862 & -1.9300 & -2.0340 & -0.6920 \\
\hline Corej & & 2.3750 & 0.9694 & & & & \\
\hline LnAreaDen & & & 0.3217 & & & & \\
\hline Net ResDen & & & & & & 0.1442 & \\
\hline Value of Time & $\$ 9.65$ & $\$ 6.58$ & $\$ 0.78$ & $\$ 1.08$ & $\$ 0.36$ & $\$ 0.23$ & $\$ 0.67$ \\
\hline $\begin{array}{l}\text { Ratio of } \\
\text { Wait/IVTT }\end{array}$ & 1.57 & - & - & 2.42 & - & - & - \\
\hline $\begin{array}{l}\text { Ratio of } \\
\text { Wait/IVTT }\end{array}$ & 2.80 & - & - & 2.35 & - & - & - \\
\hline
\end{tabular}

Note: EMPD: employment density; Zero VHHD: zero vehicle per household; $\mathrm{VHH}$ : vehicle per household; $\mathrm{PHH}$ : population per household; Rurali: rural in production zone; Corej: core zone (CBD) in attraction zone; LnAeraDen: natual log of area density; Net ResDen: net residential density.

Source: Santa Clara Valley Transportation Authority, Valley Transportation Plan 2035, 2009; Transit Cooperative Research Program Report, Appendices to TCRP Report 118, 2006; VTA Model 
Table 3 shows the results of BRT constants by applying Eq. (2). Estimated BRT constant for home-base work is -0.99530 , close to the light rail constant -0.96318 . For home-based shopping, home-based social/recreation, home-based grade school, and home-based high school, light rail constant and local bus are considered as the same mode in VTA model, so that the estimated BRT constants are the same as light rail and local bus constants. For non-home-based trips, BRT constant is equal to local bus constant because BRT and local bus has the same bias time constant for non-work trips.

\section{Table 3. BRT Constant Calculation}

\begin{tabular}{|l|c|c|c|c|c|c|c|}
\hline & $\begin{array}{c}\text { Home- } \\
\text { Based } \\
\text { Work }\end{array}$ & $\begin{array}{c}\text { Home- } \\
\text { Based } \\
\text { Shopping }\end{array}$ & $\begin{array}{c}\text { Home- } \\
\text { Based } \\
\text { Social/ } \\
\text { Recreation }\end{array}$ & $\begin{array}{c}\text { Non- } \\
\text { Home } \\
\text { Based }\end{array}$ & $\begin{array}{c}\text { Home- } \\
\text { Based } \\
\text { School } \\
\text { (Grade } \\
\text { School) }\end{array}$ & $\begin{array}{c}\text { Home- } \\
\text { Based } \\
\text { School } \\
\text { (High } \\
\text { School) }\end{array}$ & $\begin{array}{c}\text { Home- } \\
\text { Based } \\
\text { School } \\
\text { (College) }\end{array}$ \\
\hline $\begin{array}{l}\text { Light Rail } \\
\text { Constant } \Delta_{\text {LRT }}\end{array}$ & -0.96318 & 1.02982 & 2.22221 & 4.84000 & 0.59115 & 1.11067 & 0.76854 \\
\hline $\begin{array}{l}\text { Local Bus } \\
\text { Constant } \Delta_{L B}\end{array}$ & -1.70196 & 1.02982 & 2.22221 & 3.57032 & 0.59115 & 1.11067 & 0.76854 \\
\hline $\begin{array}{l}\text { Light Rail Bias } \\
\text { Time } b_{L R T}\end{array}$ & 0 & 193.33 & 193.33 & 193.33 & 193.33 & 193.33 & 193.33 \\
\hline BRT Bias Time $b_{B R T}$ & 1.03 & 198.08 & 198.08 & 198.08 & 198.08 & 198.08 & 198.08 \\
\hline $\begin{array}{l}\text { Local Bus Bias } \\
\text { Time } b_{L B}\end{array}$ & 23.69 & 198.08 & 198.08 & 198.08 & 198.08 & 198.08 & 198.08 \\
\hline $\begin{array}{l}\text { Estimated BRT } \\
\text { Constant } \Delta_{B R T}\end{array}$ & -0.99530 & 1.02982 & 2.22221 & 3.57032 & 0.59115 & 1.11067 & 0.76854 \\
\hline
\end{tabular}

\section{BRT Strategic Plan}

BRT ridership estimates for VTA's BRT Strategic Plan were developed based on the results of the improved VTA model with the added BRT mode in the mode choice model. Eleven different BRT alternatives and operating and infrastructure strategies were proposed. Six potential BRT corridors were identified by the recent Comprehensive Operations Analysis and from VTA's Long-Range Countywide Transportation Plan (Valley Transportation Plan 2035) (VTA 2009), and these included the Alum Rock, El Camino, King Road, Monterey Highway, Stevens Creek, and Sunnyvale-Cupertino BRT corridors, all shown in Figure 2. Six lines show the potential BRT corridors, which are not covered by the LRT. An assessment of new 
BRT services was conducted on three corridors within the county as the most promising alignments for near-term BRT implementation. The three corridors included:

- Alum Rock-stretching from HP Pavilion to Eastridge Mall (6.9 miles) and currently served by Rapid 522 (15-minute headways), Local Route 22 (12-minute headways), and Local Route 23 (12-minute headways).

- El Camino-stretching from Palo Alto Transit Center to HP Pavilion (16.6 miles) and currently served by Rapid 522 (15-minute headways) and Local Route 22 (12-minute headways).

- Stevens Creek-stretching from De Anza College to Downtown San Jose (8.6 miles) and currently served by Local Route 23 (12-minute headways).

Rapid 522 has the same route alignment as Local Route 22 with less headway but longer stop spacing. In the previous model, all Rapid 522, Local Route 22, and Local Route 23 are considered as local bus mode. The operating plan in these three corridors is shown in Figure 3.

Two new BRT services were proposed in these three corridors: BRT 522 to replace Rapid 522 and overlay on the Local Route 22, and BRT 523 to overlay and complement Local Route 23. Eleven operating plans were developed seeking to achieve enhanced transit market share in the corridor, while making transit more efficient and effective at serving riders. The No Project and 10 operating plans were proposed based on different combinations of BRT and local bus service areas and headways. Note that:

(1) Option 6 considers BRT 522 and 523 modeled as an LRT mode using Option 4 as a base.

(2) BRT 522 in the No Project is the existing Rapid 522. The existing Rapid 522 currently provides 15 -minute headways and fewer bus stops than Local Route 22 and is considered as a local bus in the previous VTA model;

(3) BRT would operate a premium service with 10-minute headways.

(4) Local Route 22 service would be fixed at 15 -minutes, a slight reduction in service from existing 12-minute, and Local Route 23 service would have a variable headway (between 15-30 minutes) to be tested in various service scenarios to gauge its impact on demand. 


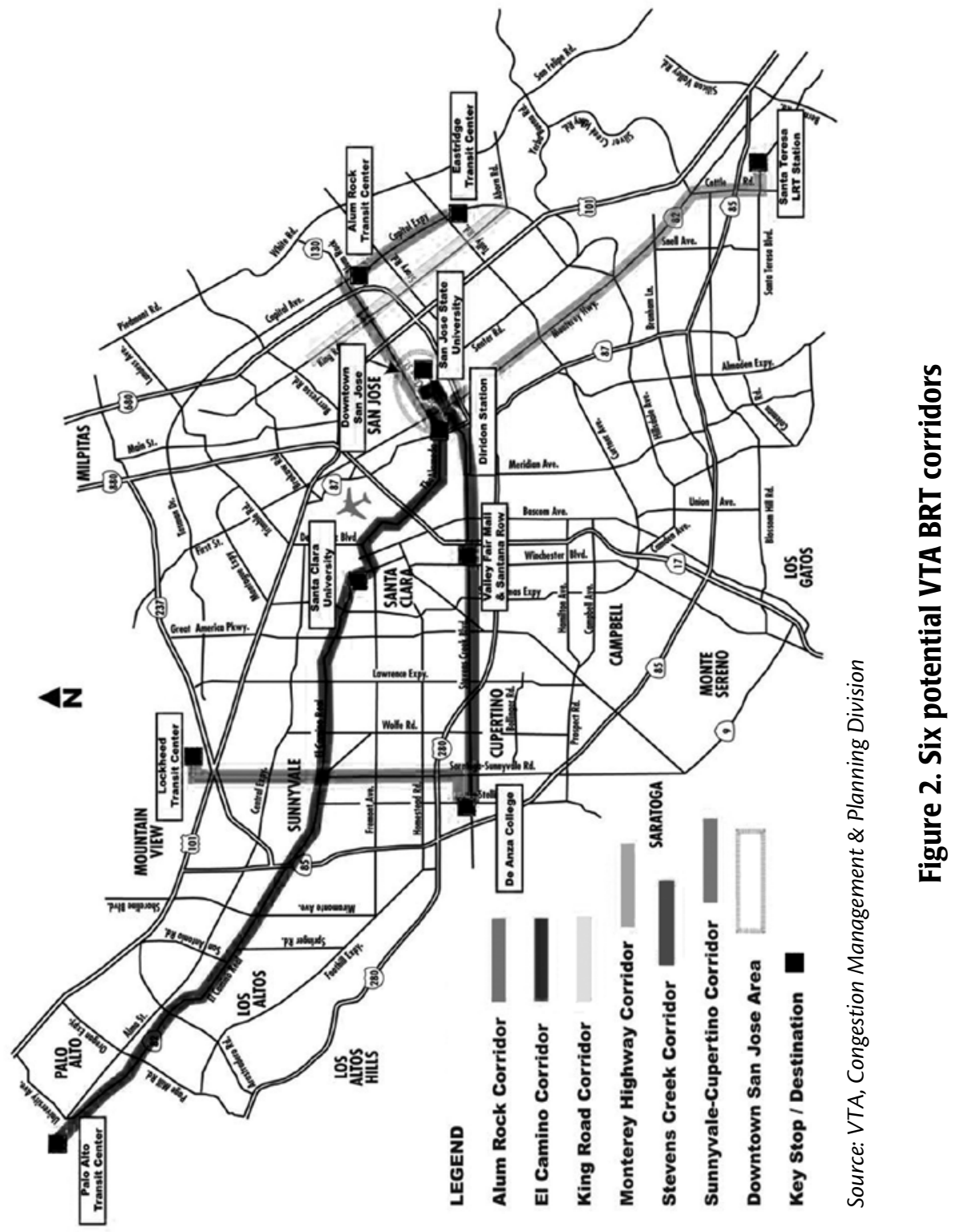




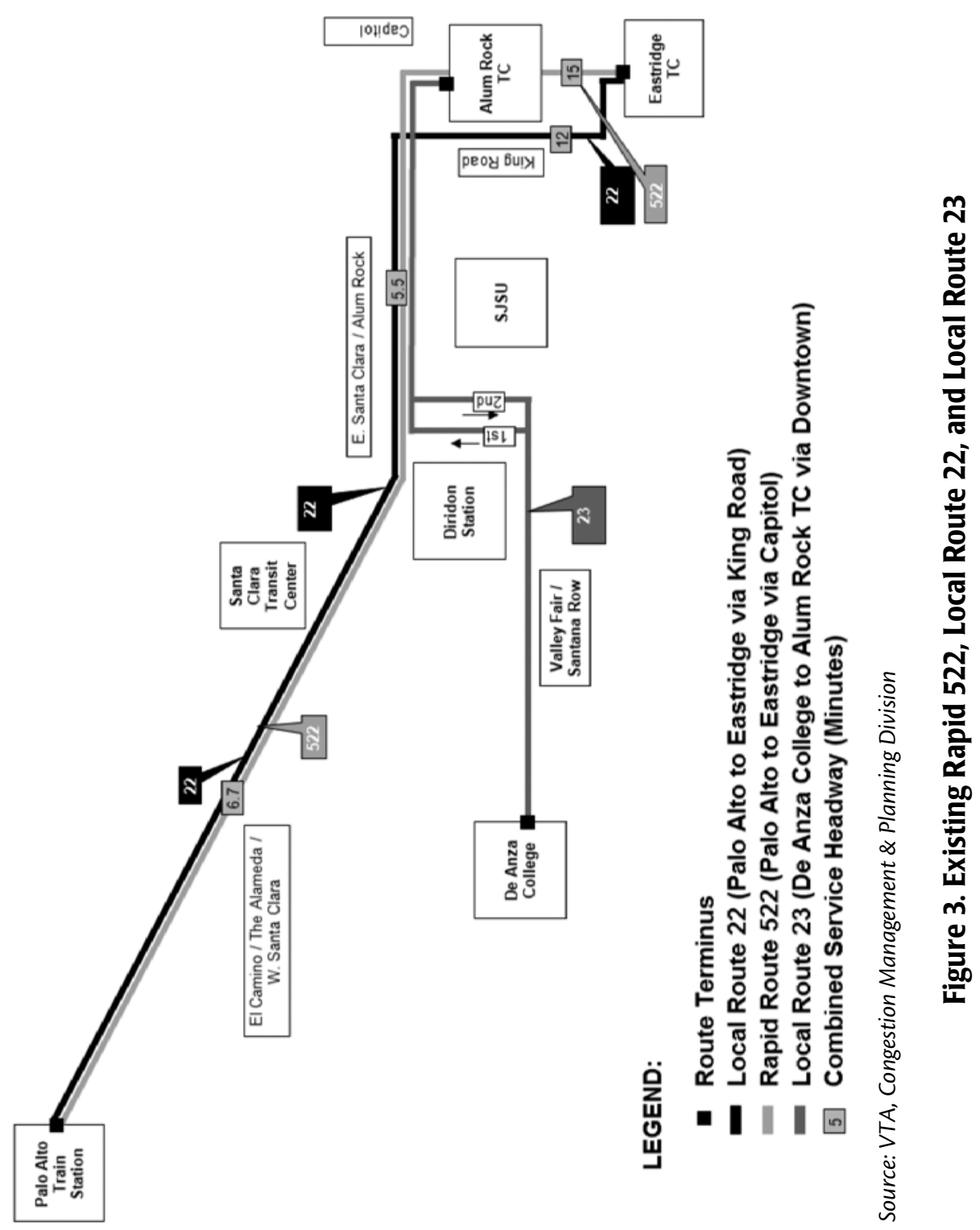


It also was assumed that in order to claim the full BRT constant, the amount of capital infrastructure required to provide the travel time savings, through either dedicated lanes with signal priority, and vehicle and station passenger amenities must be accounted for in the BRT alternative definition and costs.

Table 4 shows the No Project and 11 operating plans by different operating combinations of BRT 522, Local Route 22, BRT 523, and Local Route 23 that were modeled. Table 5 shows the 2030 boardings for the No Project and the 11 BRT operating plans. Option 6 has the highest boardings for the 522/523 BRT corridors at 91,769 daily boardings, with VTA total transit system boardings of 409,859, because BRT was assumed to have the same constant as LRT in this option plan. Option 4 modeled as a BRT mode results in 79,494 daily boardings for the 522/523 BRT corridors; this translates to a 15 percent decrease in BRT ridership if BRT is treated as a separate BRT mode and not the same as LRT. Option 4a with BRT modeled as a local bus mode results in 65,985 daily boardings for the 522/523 BRT corridor routes and 375,713 VTA total transit system boardings. This represents a 17 percent decrease in BRT ridership over the BRT constant model if BRT is treated as a local bus mode.

Table 4. No Project and Eleven BRT Operating Plans

\begin{tabular}{|l|l|l|l|l|}
\hline & BRT Route 522 & Local Route 22 & BRT Route 523 & Local Route 23 \\
\hline No Project & $\begin{array}{l}\text { Rapid, Palo Alto } \\
\text { to Eastridge via } \\
\text { Capitol (15-min } \\
\text { headways) }\end{array}$ & $\begin{array}{l}\text { Palo Alto to } \\
\text { Eastridge via King } \\
\text { Road (12-min } \\
\text { headways }\end{array}$ & N/A & $\begin{array}{l}\text { De Anza College } \\
\text { to Alum Rock via } \\
\text { Downtown (30- } \\
\text { min headways) }\end{array}$ \\
\hline Option 1 & $\begin{array}{l}\text { Palo Alto to East- } \\
\text { ridge via Capitol } \\
\text { (10-min headways) }\end{array}$ & $\begin{array}{l}\text { Palo Alto to } \\
\text { Eastridge via King } \\
\text { Road (15-min } \\
\text { headways) }\end{array}$ & $\begin{array}{l}\text { Valley Fair/Santana } \\
\text { Row to Eastridge via } \\
\text { Downtown/Capitol } \\
\text { (10-min headways) }\end{array}$ & $\begin{array}{l}\text { De Anza Col- } \\
\text { lege to SJSU via } \\
\text { Downtown (30- } \\
\text { min headways) }\end{array}$ \\
\hline Option 2 & $\begin{array}{l}\text { Palo Alto to East- } \\
\text { ridge via Capitol } \\
\text { (10-min headways) }\end{array}$ & $\begin{array}{l}\text { Palo Alto to } \\
\text { Eastridge via King } \\
\text { Road (15-min } \\
\text { headways) }\end{array}$ & $\begin{array}{l}\text { Valley Fair/Santana } \\
\text { Row to Eastridge via } \\
\text { SJSU/Capitol (10- } \\
\text { min headways) }\end{array}$ & $\begin{array}{l}\text { De Anza Col- } \\
\text { lege to SJSU via } \\
\text { Downtown (30- } \\
\text { min headways) }\end{array}$ \\
\hline Option 3a & $\begin{array}{l}\text { Palo Alto to SJSU } \\
\text { via Downtown } \\
\text { (10-min headways) }\end{array}$ & $\begin{array}{l}\text { Palo Alto to } \\
\text { Eastridge via King } \\
\text { Road (15-min } \\
\text { headways) }\end{array}$ & $\begin{array}{l}\text { Valley Fair/Santana } \\
\text { Row to Eastridge via } \\
\text { Downtown/Capitol } \\
\text { (10-min headways) }\end{array}$ & $\begin{array}{l}\text { De Anza College } \\
\text { to Alum Rock via } \\
\text { Downtown (30- } \\
\text { min headways) }\end{array}$ \\
\hline
\end{tabular}


Table 4. No Project and Eleven BRT Operating Plans (cont'd)

\begin{tabular}{|c|c|c|c|c|}
\hline Option 3b & $\begin{array}{l}\text { Palo Alto to SJSU } \\
\text { via Downtown } \\
\text { (10-min headways) }\end{array}$ & $\begin{array}{l}\text { Palo Alto to } \\
\text { Eastridge via King } \\
\text { Road (15-min } \\
\text { headways) }\end{array}$ & $\begin{array}{l}\text { De Anza College } \\
\text { to Eastridge via } \\
\text { Downtown/Capitol } \\
\text { (10-min headways) }\end{array}$ & $\begin{array}{l}\text { De Anza College } \\
\text { to Alum Rock via } \\
\text { Downtown (30- } \\
\text { min headways) }\end{array}$ \\
\hline $\begin{array}{l}\text { Option } 4 \\
\text { (modeled } \\
\text { as BRT) }\end{array}$ & $\begin{array}{l}\text { Palo Alto to East- } \\
\text { ridge via Capitol } \\
\text { (10-min headways) }\end{array}$ & $\begin{array}{l}\text { Palo Alto to } \\
\text { Eastridge via King } \\
\text { Road (15-min } \\
\text { headways) }\end{array}$ & $\begin{array}{l}\text { De Anza College } \\
\text { to Eastridge via } \\
\text { Downtown/Capitol } \\
\text { (10-min headways) }\end{array}$ & N/A \\
\hline $\begin{array}{l}\text { Option 4a* } \\
\text { (modeled } \\
\text { as Local } \\
\text { Bus) }\end{array}$ & $\begin{array}{l}\text { Palo Alto to East- } \\
\text { ridge via Capitol } \\
\text { (10-min headways) }\end{array}$ & $\begin{array}{l}\text { Palo Alto to } \\
\text { Eastridge via King } \\
\text { Road (15-min } \\
\text { headways) }\end{array}$ & $\begin{array}{l}\text { De Anza College } \\
\text { to Eastridge via } \\
\text { Downtown/Capitol } \\
\text { (10-min headways) }\end{array}$ & N/A \\
\hline Option 5 & $\begin{array}{l}\text { Palo Alto to East- } \\
\text { ridge via Capitol } \\
\text { (10-min headways) }\end{array}$ & $\begin{array}{l}\text { Palo Alto to } \\
\text { Eastridge via King } \\
\text { Road (15-min } \\
\text { headways) }\end{array}$ & $\begin{array}{l}\text { Valley Fair/Santana } \\
\text { Row to Eastridge via } \\
\text { Downtown/Capitol } \\
\text { (10-min headways) }\end{array}$ & $\begin{array}{l}\text { De Anza Col- } \\
\text { lege to SJSU via } \\
\text { Downtown ( } 30- \\
\text { min headways) }\end{array}$ \\
\hline $\begin{array}{l}\text { Option 6** } \\
\text { (modeled } \\
\text { as LRT) }\end{array}$ & $\begin{array}{l}\text { Palo Alto to East- } \\
\text { ridge via Capitol } \\
\text { (10-min headways) }\end{array}$ & $\begin{array}{l}\text { Palo Alto to } \\
\text { Eastridge via King } \\
\text { Road (15-min } \\
\text { headways) }\end{array}$ & $\begin{array}{l}\text { De Anza College } \\
\text { to Eastridge via } \\
\text { Downtown/Capitol } \\
\text { (10-min headways) }\end{array}$ & N/A \\
\hline $\begin{array}{l}\text { Option } 7 \\
\text { (BRT 10-20) }\end{array}$ & $\begin{array}{l}\text { Palo Alto to East- } \\
\text { ridge via Capitol } \\
\text { (10-min headways) }\end{array}$ & $\begin{array}{l}\text { Palo Alto to } \\
\text { Eastridge via King } \\
\text { Road (15-min } \\
\text { headways) }\end{array}$ & $\begin{array}{l}\text { De Anza College } \\
\text { to Eastridge via } \\
\text { Downtown/Capitol } \\
\text { (10-min headways) }\end{array}$ & $\begin{array}{l}\text { De Anza Col- } \\
\text { lege to SJSU via } \\
\text { Downtown (20- } \\
\text { min headways) }\end{array}$ \\
\hline $\begin{array}{l}\text { Option 7a } \\
\text { (BRT 10-15) }\end{array}$ & $\begin{array}{l}\text { Palo Alto to East- } \\
\text { ridge via Capitol } \\
\text { (10-min headways) }\end{array}$ & $\begin{array}{l}\text { Palo Alto to } \\
\text { Eastridge via King } \\
\text { Road (15-min } \\
\text { headways) }\end{array}$ & $\begin{array}{l}\text { De Anza College } \\
\text { to Eastridge via } \\
\text { Downtown/Capitol } \\
\text { (10-min headways) }\end{array}$ & $\begin{array}{l}\text { De Anza Col- } \\
\text { lege to SJSU via } \\
\text { Downtown (15- } \\
\text { min headways) }\end{array}$ \\
\hline $\begin{array}{l}\text { Option 7b } \\
\text { (BRT 10-30) }\end{array}$ & $\begin{array}{l}\text { Palo Alto to East- } \\
\text { ridge via Capitol } \\
\text { (10-min headways) }\end{array}$ & $\begin{array}{l}\text { Palo Alto to } \\
\text { Eastridge via King } \\
\text { Road (15-min } \\
\text { headways) }\end{array}$ & $\begin{array}{l}\text { De Anza College } \\
\text { to Eastridge via } \\
\text { Downtown/Capitol } \\
\text { (10-min headways) }\end{array}$ & $\begin{array}{l}\text { De Anza Col- } \\
\text { lege to SJSU via } \\
\text { Downtown (30- } \\
\text { min headways) }\end{array}$ \\
\hline
\end{tabular}

Note: * Option $4 a$ considers BRT 522 and 523 as Local Bus mode using Option 4 as the base.

** Option 6 considers BRT 522 and 523 as LRT mode using Option 4 as the base. 


\begin{tabular}{|c|c|c|c|c|c|c|c|c|c|c|c|}
\hline $\begin{array}{l}\stackrel{0}{\Lambda} \\
\text { ôn }\end{array}$ & $\begin{array}{l}\infty \\
\infty \\
\hat{0} \\
\stackrel{N}{-1}\end{array}$ & $\begin{array}{l}\overline{0} \\
\vdots \\
i\end{array}$ & 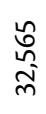 & 号 & $\begin{array}{l}n \\
0 \\
\infty \\
\infty^{\circ}\end{array}$ & $\begin{array}{l}\text { U. } \\
\infty \\
\infty \\
\infty \\
\infty\end{array}$ & $\begin{array}{l}\stackrel{m}{c} \\
\frac{m}{E}\end{array}$ & 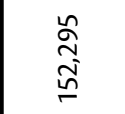 & \begin{tabular}{l}
$\infty$ \\
$\vdots$ \\
\multirow{\sim}{*}{}
\end{tabular} & 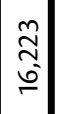 & 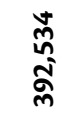 \\
\hline مّ & 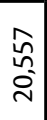 & $\underset{\sigma}{\stackrel{N}{f}}$ & $\begin{array}{l}\tilde{N} \\
\hat{n} \\
\tilde{n}\end{array}$ & 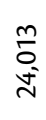 & 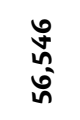 & $\begin{array}{l}\hat{N} \\
\hat{n} \\
\hat{\infty}\end{array}$ & 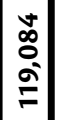 & 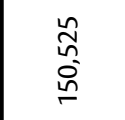 & $\begin{array}{l}\stackrel{\infty}{\infty} \\
\infty \\
\stackrel{\sim}{\sim}\end{array}$ & 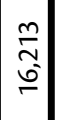 & 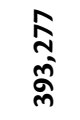 \\
\hline ठี้ & $\begin{array}{l}\hat{0} \\
\stackrel{0}{0} \\
0\end{array}$ & 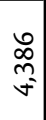 & $\begin{array}{l}\stackrel{a}{\mathcal{W}} \\
\stackrel{2}{n} \\
\tilde{m}\end{array}$ & \begin{tabular}{l}
$\stackrel{ \pm}{N}$ \\
$\infty$ \\
\multirow{\sim}{*}{}
\end{tabular} & $\stackrel{\substack{\infty \\
\\
\tilde{n}}}{n}$ & 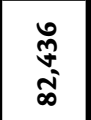 & $\begin{array}{l}\stackrel{ }{N} \\
\stackrel{n}{=}\end{array}$ & 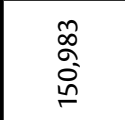 & $\begin{array}{l}\tilde{\tilde{n}} \\
\text { }\end{array}$ & \begin{tabular}{l}
0 \\
\multirow{\sigma}{\sigma}{} \\
$\stackrel{0}{0}$
\end{tabular} & 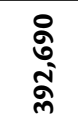 \\
\hline $\begin{array}{l}\stackrel{0}{0} \\
0\end{array}$ & $\begin{array}{l}\text { ర్ } \\
\tilde{\sigma} \\
\sigma\end{array}$ & 0 & 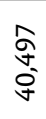 & $\frac{}{}$ & 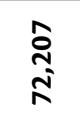 & $\begin{array}{l}\stackrel{8}{5} \\
\frac{5}{\sigma}\end{array}$ & $\begin{array}{l}\infty \\
\hat{n} \\
\tilde{n} \\
\stackrel{n}{\sim}\end{array}$ & $\begin{array}{l}\text { 今 } \\
\infty \\
\text { กิ } \\
\end{array}$ & 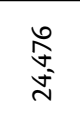 & 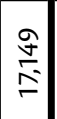 & 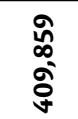 \\
\hline$\stackrel{n}{艹}$ & $\begin{array}{l}\bar{n} \\
\stackrel{-}{0} \\
\end{array}$ & $\underset{\sigma}{\stackrel{\infty}{6}}$ & 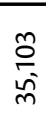 & $\begin{array}{l}\frac{n}{\sigma} \\
\stackrel{5}{n}\end{array}$ & 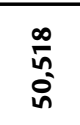 & $\begin{array}{l}\text { f } \\
\stackrel{\infty}{\wedge} \\
\stackrel{1}{n}\end{array}$ & $\begin{array}{l}\infty \\
\stackrel{\infty}{0} \\
0 \\
\sigma \\
=\end{array}$ & $\begin{array}{l}\tilde{\widetilde{\sigma}} \\
\stackrel{\tilde{n}}{n}\end{array}$ & $\begin{array}{l}\hat{\curvearrowright} \\
\text { } \\
\text { }\end{array}$ & $\begin{array}{c}\checkmark \\
\tilde{m} \\
\sigma \\
\sigma\end{array}$ & 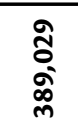 \\
\hline $\begin{array}{l}\text { fo } \\
\stackrel{0}{0} \\
\text { o }\end{array}$ & 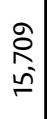 & 0 & 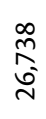 & $\begin{array}{l}\stackrel{\infty}{N} \\
\stackrel{n}{n} \\
\stackrel{n}{N}\end{array}$ & 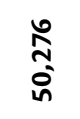 & 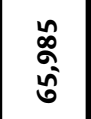 & 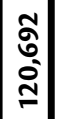 & 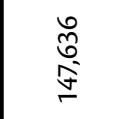 & $\begin{array}{l}\mathscr{0} \\
\infty \\
0 \\
\mathbb{N}\end{array}$ & $\frac{n}{m}$ & $\underset{n}{\stackrel{m}{n}}$ \\
\hline ơ & 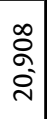 & 0 & \begin{tabular}{l}
$\infty$ \\
\multirow{0}{0}{} \\
$\tilde{N}$ \\
$\tilde{n}$
\end{tabular} & $\begin{array}{l}\infty \\
0 \\
\text { ర్ }\end{array}$ & 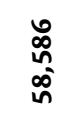 & 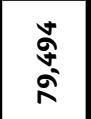 & 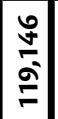 & $\begin{array}{l}\stackrel{N}{n} \\
\stackrel{N}{n} \\
\end{array}$ & $\begin{array}{l}\stackrel{8}{\circ} \\
\stackrel{ \pm}{*}\end{array}$ & 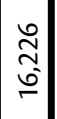 & 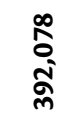 \\
\hline مै & $\begin{array}{l}\stackrel{m}{\infty} \\
\stackrel{m}{n} \\
\stackrel{\sim}{n}\end{array}$ & $\stackrel{n}{\stackrel{n}{N}}$ & $\begin{array}{l}\bar{\sigma} \\
\text { సे }\end{array}$ & 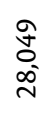 & 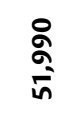 & $\begin{array}{l}\infty \\
\infty \\
0 \\
0 \\
\end{array}$ & 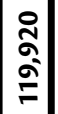 & $\begin{array}{l}\stackrel{n}{8} \\
\stackrel{0}{n} \\
\stackrel{5}{n}\end{array}$ & $\begin{array}{l}\hat{\circ} \\
\text { ஸे }\end{array}$ & 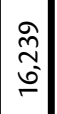 & 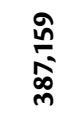 \\
\hline $\begin{array}{l}\tilde{n} \\
\stackrel{n}{0} \\
0\end{array}$ & $\begin{array}{l}\stackrel{m}{m} \\
\stackrel{\sim}{\sim}\end{array}$ & 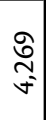 & 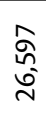 & \begin{tabular}{l}
$\stackrel{ి}{6}$ \\
\multirow{+}{\infty}{} \\
$\infty$
\end{tabular} & 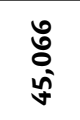 & $\begin{array}{l}\stackrel{\infty}{\circ} \\
\text { م્ } \\
\text { م }\end{array}$ & $\begin{array}{l}\hat{n} \\
\hat{\sigma} \\
\underline{F}\end{array}$ & $\begin{array}{l}\stackrel{\infty}{\sigma} \\
\stackrel{\sim}{n}\end{array}$ & 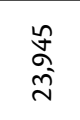 & $\begin{array}{c}\hat{\tilde{m}} \\
\tilde{\sigma} \sigma \\
\underline{\sigma}\end{array}$ & 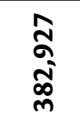 \\
\hline ثّم & $\begin{array}{l}\hat{b} \\
\vdots \\
\grave{\sim}\end{array}$ & 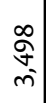 & 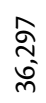 & $\stackrel{\infty}{\stackrel{\infty}{\simeq}}$ & \begin{tabular}{l}
$n$ \\
$\hat{n}$ \\
$\infty$ \\
\multirow{\sigma}{*}{}
\end{tabular} & 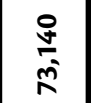 & $\begin{array}{l}\bar{N} \\
\sigma \\
\bar{E}\end{array}$ & $\begin{array}{l}\infty \\
\hat{0} \\
\hat{n} \\
\end{array}$ & \begin{tabular}{l} 
ণ \\
\multirow{+}{*}{} \\
\multirow{d}{*}{}
\end{tabular} & 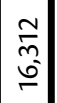 & 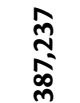 \\
\hline పัँ & $\begin{array}{l}\text { o } \\
\stackrel{2}{-} \\
\stackrel{\nu}{-1}\end{array}$ & $\begin{array}{l}\hat{a} \\
\stackrel{+}{m}\end{array}$ & $\begin{array}{l}\stackrel{\sigma}{\sigma} \\
\stackrel{n}{m}\end{array}$ & $\begin{array}{l}\infty \\
\stackrel{0}{0} \\
\stackrel{n}{n} \\
\sim\end{array}$ & $\begin{array}{l}\hat{y} \\
\text { ñ } \\
\text { ñ }\end{array}$ & 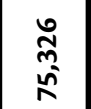 & $\begin{array}{l}\text { ○ } \\
\stackrel{\alpha}{ } \\
\infty \\
=\end{array}$ & 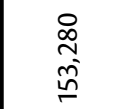 & 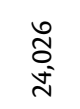 & $\begin{array}{c}\tilde{n} \\
\underset{n}{\sigma} \\
\sigma \\
\sigma\end{array}$ & $\begin{array}{l}\overline{0} \\
\stackrel{\infty}{\infty} \\
\stackrel{0}{\infty}\end{array}$ \\
\hline 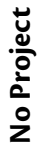 & 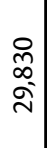 & $\begin{array}{l}\stackrel{0}{\circ} \\
\text { ఠ̆ }\end{array}$ & 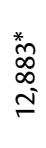 & 0 & $\begin{array}{l}\infty \\
\infty \\
\infty \\
\simeq \\
\simeq\end{array}$ & 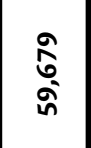 & 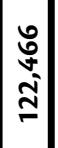 & \begin{tabular}{l}
$\stackrel{\infty}{\hat{n}}$ \\
$\tilde{n}$ \\
\multirow{v}{*}{}
\end{tabular} & $\begin{array}{l}\stackrel{0}{\sigma} \\
\stackrel{\sim}{\sim}\end{array}$ & 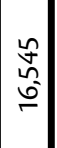 & $\underset{\substack{i\\
}}{\infty}$ \\
\hline 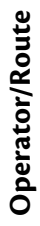 & 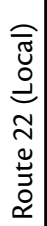 & 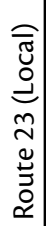 & 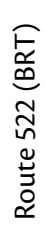 & 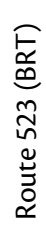 & 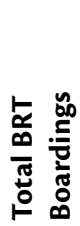 & 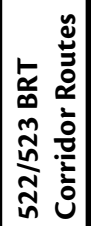 & 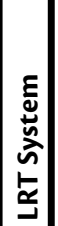 & 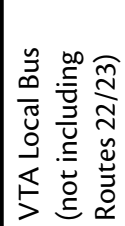 & 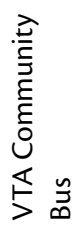 & 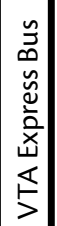 & 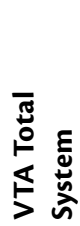 \\
\hline
\end{tabular}


The ultimate preferred BRT Option 7a has the second highest boardings for the $522 / 523$ BRT corridors at 83,577 daily boardings, with VTA total transit system boardings of 393,277 , by using the BRT constants derived from Table 3 in the improved VTA model. Option 7a also would generate the second largest total new transit trips, including home-based work and non-work trips, as shown in Table 6. The potential new transit riders would be up to 36 percent of BRT ridership in the preferred operating plan Option 7a, which is a little higher than the 24 to 33 percent from the FTA's study of BRT systems currently in operation (Peak et al. 2005).

The operating costs and capital costs for the 11 BRT operating plans are listed in Table 7. Detailed operating and capital cost analysis can be found in the VTA BRT Strategic Plan (2009). Without considering Option 6 (BRT treated as LRT mode), after demand, operating cost, and capital cost analysis, Option 7a was selected as the preferred BRT operating plan, which would generate the highest demand and the largest number of new riders, but include the highest operating costs as well. The operating and routing plan of Option $7 \mathrm{a}$ is shown in Figure 4.

\section{Conclusions}

A state-of-the-practice travel demand model with a new BRT mode included in the mode choice model was developed by the Santa Clara VTA and now is used in planning and design phases for countywide BRT projects. Instead of considering BRT the same as LRT or local bus, the BRT constants derived from the Market Research Model fall between LRT and local bus constants. The application of the BRT constants results in BRT ridership between ridership estimates prepared with BRT having a local bus constant and for BRT having a LRT constant, with a variation of approximately 15 percent higher or lower, depending on which constant BRT employed in the forecasts. The improved VTA model was expected to forecast more reasonable future BRT boardings, which were an important consideration in light of the relatively high capital and operating costs associated with BRT services. The potential new transit riders after BRT lines open would be up to 36 percent of BRT ridership in the preferred operating plan.

Future extensions of the present work might include developing a peer review of before-and-after BRT implementation studies and an evaluation of how actual ridership compares to forecasted ridership for areas implementing BRT, either through passenger counts or on-board surveys reflecting the situation at least one year after BRT lines opens. The Alum Rock segment of the BRT lines 522/523 is currently in final design and scheduled for completion by 2013. The remainder 
Journal of Public Transportation, Vol. 14, No. 3, 2011

\begin{tabular}{|c|c|c|c|c|c|c|c|}
\hline $\begin{array}{l}\stackrel{0}{\hat{n}} \\
\stackrel{\circ}{0}\end{array}$ & $\begin{array}{l}\hat{\tilde{N}} \\
\stackrel{\sigma}{\sigma}\end{array}$ & $\begin{array}{l}\text { 市 } \\
\infty \\
\stackrel{\infty}{\sim}\end{array}$ & $\begin{array}{l}\stackrel{v}{b} \\
\infty \\
\infty \\
m\end{array}$ & $\begin{array}{l}\hat{\tilde{n}} \\
\text { iे }\end{array}$ & 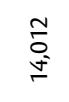 & 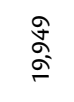 & $\begin{array}{l}\stackrel{\circ}{+} \\
\underset{m}{*}\end{array}$ \\
\hline $\begin{array}{l}\text { م⿱ } \\
\stackrel{\circ}{0}\end{array}$ & $\begin{array}{l}\tilde{N} \\
\infty \\
\tilde{\sigma} \\
\tilde{\sigma}\end{array}$ & 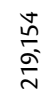 & $\begin{array}{l}\stackrel{\circ}{\circ} \\
\stackrel{\infty}{\infty} \\
\stackrel{m}{m}\end{array}$ & 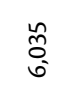 & 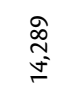 & 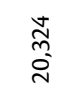 & $\begin{array}{l}\stackrel{\circ}{\circ} \\
\text { ஸे }\end{array}$ \\
\hline ڤ̆ & $\begin{array}{l}\stackrel{\checkmark}{\nwarrow} \\
\stackrel{\sigma}{\sigma}\end{array}$ & $\frac{\hat{O}}{\bar{\sigma}}$ & $\begin{array}{l}\bar{\circ} \\
\stackrel{\alpha}{ } \\
\stackrel{m}{m}\end{array}$ & $\begin{array}{l}\text { ू̆ } \\
\text { जे }\end{array}$ & $\underset{\underset{\sim}{\mathbb{J}}}{\stackrel{\mathcal{V}}{\sim}}$ & $\begin{array}{l}\stackrel{\sim}{N} \\
\stackrel{\sim}{\sim}\end{array}$ & 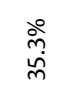 \\
\hline $\begin{array}{l}\text { ○̆ } \\
\text { ŏ }\end{array}$ & 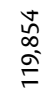 & $\begin{array}{l}\stackrel{ \pm}{N} \\
\underset{\sim}{\mathbb{N}}\end{array}$ & 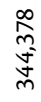 & $\begin{array}{l}\text { 岂 } \\
\text { o } \\
0\end{array}$ & $\begin{array}{l}\hat{\widehat{C}} \\
\text { مू }\end{array}$ & $\stackrel{n}{\stackrel{n}{\sim}}$ & $\begin{array}{l}\stackrel{\circ}{0} \\
\text { ஸे }\end{array}$ \\
\hline$\stackrel{n}{\tilde{0}}$ & $\begin{array}{l}\underbrace{+} \\
\alpha \\
\infty \\
\rightleftharpoons\end{array}$ & $\begin{array}{l}\underset{N}{N} \\
\underset{\sigma}{N} \\
\grave{N}\end{array}$ & 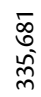 & $\begin{array}{l}\frac{5}{h} \\
\frac{5}{n}\end{array}$ & $\begin{array}{l}\mathbb{S} \\
\infty \\
=\end{array}$ & $\begin{array}{l}0 \\
\stackrel{0}{\circ} \\
\therefore\end{array}$ & 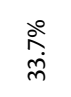 \\
\hline 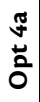 & 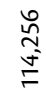 & 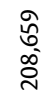 & $\begin{array}{l}\frac{n}{\sigma} \\
\underset{n}{\tilde{n}}\end{array}$ & 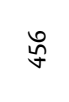 & 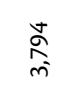 & \begin{tabular}{l}
$\stackrel{0}{N}$ \\
\multirow{v}{*}{}
\end{tabular} & 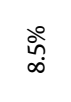 \\
\hline$\stackrel{v}{\stackrel{ \pm}{0}}$ & $\begin{array}{l}\infty \\
\stackrel{0}{6} \\
\stackrel{2}{E}\end{array}$ & $\begin{array}{l}\text { N } \\
\hat{\alpha} \\
\infty \\
\sim\end{array}$ & $\begin{array}{l}\stackrel{\circ}{\circ} \\
\infty \\
m \\
m\end{array}$ & 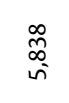 & $\begin{array}{l}\hat{\infty} \\
0 \\
\stackrel{m}{\sim} \\
\end{array}$ & $\begin{array}{l}\tilde{N} \\
\tilde{\tilde{\Omega}}\end{array}$ & 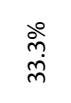 \\
\hline $\begin{array}{l}\hat{m} \\
\stackrel{\tilde{a}}{0}\end{array}$ & $\begin{array}{l}\hat{\circ} \\
\stackrel{0}{\check{E}}\end{array}$ & 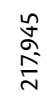 & $\underset{m}{\tilde{c}}$ & $\begin{array}{l}\hat{D} \\
\text { in }\end{array}$ & $\begin{array}{l}\text { o } \\
0 \\
\stackrel{0}{m}\end{array}$ & 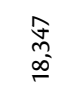 & 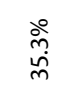 \\
\hline $\begin{array}{l}\stackrel{m}{ٌ} \\
\stackrel{0}{0}\end{array}$ & $\stackrel{\stackrel{్}{m}}{\stackrel{\infty}{\rightleftharpoons}}$ & $\begin{array}{l}\text { N్} \\
\stackrel{n}{n} \\
\stackrel{n}{N}\end{array}$ & 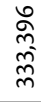 & 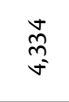 & 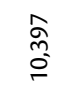 & 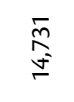 & $\begin{array}{l}\stackrel{\circ}{\hat{N}} \\
\text { m. }\end{array}$ \\
\hline ثّ̊ & 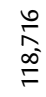 & $\begin{array}{l}\stackrel{\infty}{N} \\
\underset{\sigma}{N} \\
\underset{N}{N}\end{array}$ & 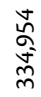 & $\underset{\sigma}{\stackrel{\sigma}{\sigma}}$ & 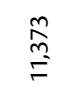 & 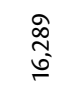 & $\begin{array}{l}\stackrel{\circ}{n} \\
\text { mn }\end{array}$ \\
\hline б̆ & $\begin{array}{l}\stackrel{\varrho}{\infty} \\
\stackrel{\infty}{\infty} \\
\stackrel{\infty}{\rightleftharpoons}\end{array}$ & $\begin{array}{l}\underset{N}{\sim} \\
\underset{\sigma}{N}\end{array}$ & 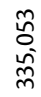 & $\begin{array}{l}0 \\
\bar{o} \\
i n\end{array}$ & $\begin{array}{l}\stackrel{\Omega}{\mathrm{m}} \\
\stackrel{\mathrm{F}}{F}\end{array}$ & $\begin{array}{l}\infty \\
\infty \\
m_{0}^{2} \\
\sigma^{0}\end{array}$ & $\underset{\text { ì }}{\stackrel{\circ}{\grave{m}}}$ \\
\hline 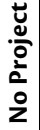 & $\begin{array}{l}\stackrel{8}{\infty} \\
\infty \\
\stackrel{\infty}{=}\end{array}$ & 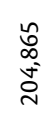 & $\begin{array}{l}\tilde{0} \\
0 \\
\infty \\
\infty \\
\infty\end{array}$ & & & & \\
\hline 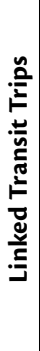 & 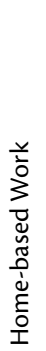 & 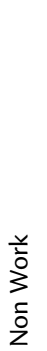 & $\begin{array}{l}\overline{0} \\
\stackrel{0}{\circ}\end{array}$ & 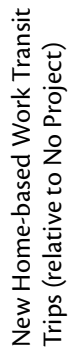 & 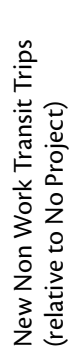 & 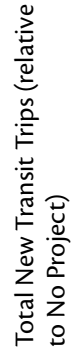 & 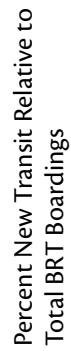 \\
\hline
\end{tabular}


of the BRT 522 corridor along El Camino Real is scheduled for completion by 2015. Based on this schedule, it is expected that the VTA will be able to implement BRT in the county within three years, which will provide an opportunity to refine the BRT models in the relative near term and develop before and after studies of actual local experiences.

Table 7. Annual Operating and Maintenance Costs and Capital Costs for Eleven BRT Operating Plans

\begin{tabular}{|l|c|c|}
\hline & $\begin{array}{c}\text { Annual Operating and } \\
\text { Maintenance Cost }\end{array}$ & Capital Cost \\
\hline No Project & - & - \\
\hline Option 1 & $\$ 62,700,000$ & $\$ 412,200,000$ \\
\hline Option 2 & $\$ 62,600,000$ & $\$ 420,900,000$ \\
\hline Option 3a & $\$ 58,900,000$ & $\$ 417,900,000$ \\
\hline Option 3b & $\$ 64,600,000$ & $\$ 495,700,000$ \\
\hline Option 4 & $\$ 64,400,000$ & $\$ 490,000,000$ \\
\hline Option 4a & $\$ 64,400,000$ & $\$ 490,000,000$ \\
\hline Option 5 & $\$ 64,700,000$ & $\$ 412,200,000$ \\
\hline Option 6 & $\$ 64,400,000$ & $\$ 490,000,000$ \\
\hline Option 7 (BRT 10-20) & $\$ 70,400,000$ & $\$ 490,000,000$ \\
\hline Option 7a (BRT 10-15) & $\$ 72,300,000$ & $\$ 490,000,000$ \\
\hline Option 7b (BRT 10-30) & $\$ 68,400,000$ & $\$ 490,000,000$ \\
\hline Option 7b (BRT 10-30) & $\$ 68,400,000$ & $\$ 490,000,000$ \\
\hline
\end{tabular}

Source: VTA BRT Strategic Plan, 2009. 
Journal of Public Transportation, Vol. 14, No. 3, 2011

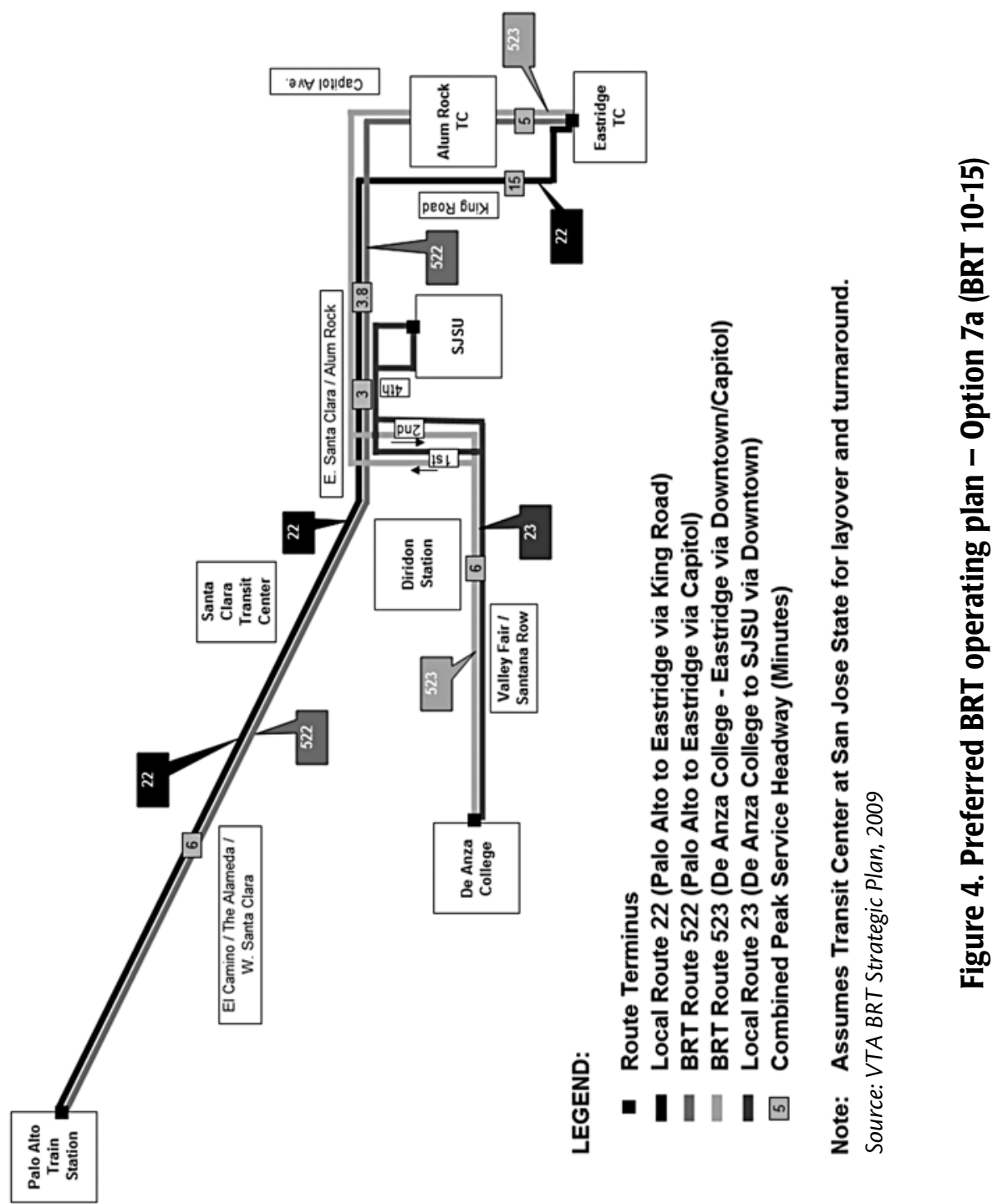




\section{References}

Levinson, H. S., S. Zimmerman, J. Clinger, and Rutherford, C. S. 2002. Bus rapid transit: An overview. Journal of Public Transportation 5(2): 1-30.

Metropolitan Transportation Commission. 1997. Travel demand models for the San Francisco Bay Area (BAYCAST-90) technical summary. Oakland, California.

Peak, M., C. Henke, and Wnuk, L. 2005. Bus rapid transit ridership analysis, Federal Transit Administration, Report FTA-CA-26-7068-2004.1.

Santa Clara Valley Transportation Authority. 2001. Silicon Valley Rapid Transit Corridor MIS/EIS/EIR Deliverable \#10: Travel demand modeling methodology report. Prepared by Hexagon Transportation Consultants. San Jose, California.

Santa Clara Valley Transportation Authority. 2007. Transit market research models. Prepared by Cambridge Systematics, Inc. San Jose, California.

Santa Clara Valley Transportation Authority. 2009. BRT strategic plan - Final report. Prepared by ARUP North America Ltd. San Jose, California.

Santa Clara Valley Transportation Authority. 2009. Valley Transportation Plan 2035. San Jose, California.

Transportation Research Board of the National Academies. 2003. Transit Cooperative Research Program Report 90. Bus rapid transit - Volume 1: Case study in bus rapid transit. Transportation Research Board, Washington D.C.

Transportation Research Board of the National Academies. 2006. Transit Cooperative Research Program Report - Appendices to TCRP Report 118. Bus Rapid transit practitioner's guide, TCRP Web-Only Document 39. Transportation Research Board, Washington D.C.

Vuchic, V. R. 2002. Bus semirapid transit mode development and evaluation. Journal of Public Transportation 5(2): 71-95.

Chun-Hung Peter Chen (peter.chen@vta.org) is a Transportation Planner in the Santa Clara Valley Transportation Authority, Santa Clara County, California. He received his Ph.D. in Civil Engineering from the University of Maryland, College Park, and his M.S. and B.S. degrees in Civil Engineering from the National Taiwan University. He has been with VTA since 2007 and worked at several light rail and BRT projects. He is a registered professional engineer in the states of Washington and 
California and also has a certificate of PTOE (Professional Transportation Operations Engineer) from the Transportation Professional Certification Board Inc.

GEORGE A. NAYLOR (george.naylor@vta.org) is a Transportation Planning Manager for the Santa Clara Valley Transportation Authority and manages the travel demand modeling activities for the Authority. He received his master's degree in Urban and Regional Planning from San Jose State University and his B.S degree in Petroleum Engineering from Texas A\&M University. 